\title{
The Energy of Cayley Graphs for a Generating Subset of the Dihedral Groups
}

\author{
${ }^{1}$ Amira Fadina Ahmad Fadzil*, ${ }^{2}$ Nor Haniza Sarmin and ${ }^{3}$ Ahmad Erfanian \\ ${ }^{1,2}$ Department of Mathematical Sciences, Universiti Teknologi Malaysia \\ 81310 UTM Johor Bahru, Malaysia \\ ${ }^{3}$ Department of Pure Mathematics, Faculty of Mathematical Sciences and \\ Center of Excellence in Analysis on Algebraic Structures, \\ Ferdowsi University of Mashhad, Mashhad, Iran \\ *Corresponding author: amirafadinaahmadfadzil@yahoo.com
}

Article history

Received: 22 December 2018

Received in revised form: 17 May 2019

Accepted: 17 July 2019

Published online: 1 December 2019

\begin{abstract}
Let $G$ be a finite group and $S$ be a subset of $G$, where $S$ does not include the identity of $G$ and is inverse closed. A Cayley graph of a group $G$ with respect to the subset $S$ is a graph, where its vertices are the elements of $G$ and two vertices $a$ and $b$ are connected if $a b^{-1}$ is in the subset $S$. The energy of a Cayley graph is the sum of all absolute values of the eigenvalues of its adjacency matrix. In this paper, we consider a specific subset $S=\left\{b, a b, \ldots, a^{n-1} b\right\}$ for dihedral groups of order $2 n$, where $n \geq 3$ and find the Cayley graph with respect to the set. We also calculate the eigenvalues and compute the energy of the respected Cayley graphs. Finally, the generalization of the energy of the respected Cayley graphs is found.
\end{abstract}

Keywords Energy of graph; eigenvalues; adjacency matrix; Cayley graph; dihedral groups.

Mathematics Subject Classification 05C25, 05C50, 15A18, 65F15.

\section{Introduction}

There have been a grown of interests for many researchers to study on the energy of graph. According to Woods [1], the study on the energy of general simple graphs was first defined by Gutman in 1978 inspired from the Huckel Molecular Orbital (HMO) Theory proposed in 1930s. The Huckel Molecular Orbital Theory has been used by chemists in approximating the energies related with $\pi$-electron orbitals in conjugated hydrocarbon molecules.

In 2009, Li et al. [2] stated that in the early days, when computers were not widely available, the calculation of the HMO total $\pi$-electron energy was a serious problem. In order to overcome the difficulty, a variety of approaches have been offered to calculate the approximate calculation of the $\pi$-electron energy. Within the HMO approximation, the total energy of the $\pi$-electrons, 
denoted by $\epsilon$ is obtained by summing distinct electron energies. In conjugated hydrocarbons, the total number of $\pi$-electrons is equal to the number of vertices of the associated molecular graph. After some considerations, they arrived at the definition of the energy, which is the sum of the absolute values of the eigenvalues of the graph.

Many researchers have studied on the topic of energy of graphs. In 2004, Bapat and Pati [3] have proved that the energy of a graph is never an odd integer. Meanwhile, the properties that the energy of a graph is never the square root of an odd integer has been proven by Pirzada and Gutman [4] in 2008. There are also a few other researchers who studied specifically on the energy of unitary Cayley graphs (see [5], [6]).

Besides, there have also been many studies on the Cayley graphs for dihedral groups. In 2006, Wang and $\mathrm{Xu}$ [7] have considered the non-normal one-regular and 4-valent Cayley graphs of dihedral groups while Kwak and Oh [8] have classified the 4-valent and 6-valent one regular normal Cayley graphs of dihedral groups whose vertex stabilizers in $A u t(\Gamma)$ are cyclic.

In addition, in 2008, Kwak et al. [9] have explored on the one-regular Cayley graphs on dihedral groups of any prescribed valency. Kim et al. [10] also have studied on the Cayley graphs of dihedral groups on the classification of $p$-valent regular Cayley graphs.

The theory of graph energy has been used by chemists in approximating the energies related to $\pi$-electron orbitals in conjugated hydrocarbon. Besides its chemical applications, there are a few applications in other field of science such as in graph entropies [11], modelling of properties of proteins [12] and in the search for the genetic causes of Alzheimer disease [13].

The target of this study is to present the energy of the Cayley graphs associated to dihedral groups for the subset $S=\left\{b, a b, \ldots, a^{n-1} b\right\}$. The procedure consists of finding the elements, vertices and edges for the Cayley graphs of the dihedral groups, finding their isomorphism, building the adjacency matrix for the Cayley graph, finding the spectrum of the adjacency matrix of the graphs and lastly calculating the energy of the graphs. Some properties and general formula for the energy will also be presented at the end of the study.

\section{Preliminaries}

The followings are some definitions that are used in this work.

Definition 1 Dihedral Group [14]

If $\pi(n)$ is a regular polygon with $n$ vertices and center $O$, then the symmetry group $\sum(\pi n)$ is called the dihedral groups with $2 n$ elements, and it is denoted by $D_{2 n}$.

Definition 2 Cayley Graph of a Group [15]

Let $G$ be a finite group with identity 1 . Let $S$ be a subset of $G$ satisfying $1 \notin S$ and $S=S^{-1}$; that is, $s \in S$ if and only if $s^{-1} \in S$. The Cayley graph $C a y(G ; S)$ on $G$ with connection set $S$ is defined as follows:

- the vertices are the elements of $G$

- there is an edge joining $g$ and $h$ if and only if $h=s g$ for some $s \in S$.

The set of all Cayley graphs on $G$ is denoted by $C a y(G, S)$, where $S$ is the subset of $G$ with a certain valency, which is the order of $S$. 
Definition 3 Complete Bipartite Graph [16]

A graph $\Gamma$ is called bipartite when its vertex set can be partitioned into two disjoint parts $X_{1}, X_{2}$ such that all edges of $\Gamma$ meet both $X_{1}$ and $X_{2}$. The graph is called complete bipartite if all possible edges that join vertices from set $X_{1}$ to set $X_{2}$ are drawn.

Definition 4 Adjacency Matrix [17]

Let $\Gamma$ be a graph with $V(\Gamma)=\{1, \ldots, n\}$ and $E(\Gamma)=\left\{e_{1}, \ldots, e_{m}\right\}$. The adjacency matrix of $\Gamma$ denoted by $A(\Gamma)$ is the $n \times n$ matrix defined as follows. The rows and the columns of $A(\Gamma)$ are indexed by $V(\Gamma)$. If $i \neq j$ then the $(i, j)$-entry of $A(\Gamma)$ is 0 for vertices $i$ and $j$ nonadjacent, and the $(i, j)$-entry is 1 for $i$ and $j$ adjacent. The $(i, i)$-entry of $A(\Gamma)$ is 0 for $i=1, \ldots, n$. $A(\Gamma)$ is often simply denoted by $A$.

Definition 5 Energy of Graph [17]

For any graph $\Gamma$, the energy of the graph is defined as $\varepsilon(\Gamma)=\sum_{i=1}^{n}\left|\lambda_{i}\right|$, where $\lambda_{1}, \ldots, \lambda_{n}$ are the eigenvalues of the adjacency matrix of $\Gamma$.

Proposition 1 Spectrum of Complete Bipartite Graph [16]

Consider the undirected complete bipartite graph $K_{m, n}$. The spectrum of a complete bipartite graph is $\left\{ \pm \sqrt{m n}, 0^{m+n-2}\right\}$.

\section{Main Results}

In this section, our main results are specified in term of proposition, lemma and theorem. Proposition 1 presents the generalization of the Cayley graphs of $D_{2 n}$ with respect to the generating set $S=\left\{b, a b, \ldots, a^{n-1} b\right\}$ while Lemma 1 states the eigenvalues of the generalized Cayley graphs in Proposition 1. Then, the results on the energy of the Cayley graphs with respect to the generating set of dihedral groups are presented in Theorem 1. The findings in Proposition 1 and Lemma 1 will be used in proving Theorem 1.

Proposition 2 Let $D_{2 n}$ be the dihedral groups of order $2 n$, where $n \geq 3$ and $S=\{b, a b, \ldots$, $\left.a^{n-1} b\right\}$ be the subset of $D_{2 n}$. The Cayley graphs of $D_{2 n}$ with respect to the subset $S$,

$$
\operatorname{Cay}\left(D_{2 n},\left\{b, a b, \ldots, a^{n-1} b\right\}\right)
$$

are the complete bipartite graph $K_{n, n}$.

Proof Consider the dihedral groups $D_{2 n}$ of order $2 n$ and the Cayley graphs of $D_{2 n}$ with respect to the subset $S=\left\{b, a b, \ldots, a^{n-1} b\right\}$, denoted as $\operatorname{Cay}\left(D_{2 n},\left\{b, a b, \ldots, a^{n-1} b\right\}\right)$. Note that $|S|=n$. By the definition of Cayley graph, the vertices of $\operatorname{Cay}\left(D_{2 n},\left\{b, a b, \ldots, a^{n-1} b\right\}\right)$ are the elements of $D_{2 n}$ and there is an edge joining vertices $h$ and $g$ if and only if $h g^{-1} \in S$. Since the order of $|S|=\frac{1}{2}\left|D_{2 n}\right|$, this forms a complete bipartition of the vertex sets $S=$ $\left\{b, a b, \ldots, a^{n-1} b\right\}$ and $D_{2 n} / S=\left\{1, a, \ldots, a^{n-1}\right\}$, where all vertices from the set $S$ are connected to all vertices from the set $D_{2 n} / S$. Therefore, since the sets $S$ and $D_{2 n} / S$ are of order $n$, then $\operatorname{Cay}\left(D_{2 n},\left\{b, a b, \ldots, a^{n-1} b\right\}\right)=K_{n, n}$.

Lemma 1 Let $D_{2 n}$ be the dihedral groups of order $2 n$, where $n \geq 3$ and $S=\left\{b, a b, \ldots, a^{n-1} b\right\}$ be the subset of $D_{2 n}$. Therefore, the eigenvalues of $\operatorname{Cay}\left(D_{2 n},\left\{b, a b, \ldots, a^{n-1} b\right\}\right)=K_{n, n}$ are 0 with multiplicity $2 n-2$ and $\pm n$ with multiplicity 1. 
Proof Consider the dihedral groups $D_{2 n}$ of order $2 n$. By Proposition 2, The Cayley graphs of $D_{2 n}$ with respect to the subset $S, \operatorname{Cay}\left(D_{2 n},\left\{b, a b, \ldots, a^{n-1} b\right\}\right)$ are the complete bipartite graph $K_{n, n}$. Since the adjacency spectrum of a complete bipartite graph $K_{m, n}$ is $\left\{ \pm \sqrt{m n}, 0^{m+n-2}\right\}$, then $\operatorname{Spec}\left(K_{n, n}\right)=\left\{ \pm \sqrt{n^{2}}, 0^{2 n-2}\right\}$, which also can be written $\lambda=0$ with multiplicity $2 n-2$ and $\lambda= \pm n$ with multiplicity 1 .

Theorem 1 Let $D_{2 n}$ be the dihedral groups of order $2 n$, where $n \geq 3$ and $S=\{b, a b, \ldots$, $\left.a^{n-1} b\right\}$ be the subset of $D_{2 n}$. The energy of the Cayley graphs of $D_{2 n}$ with respect to the subset $S$,

$$
E\left(C a y\left(D_{2 n},\left\{b, a b, \ldots, a^{n-1} b\right\}\right)\right)=2 n .
$$

Proof Consider the dihedral groups $D_{2 n}$ of order $2 n$. By Proposition 2 and Lemma 1, the Cayley graphs of $D_{2 n}$ with respect to the subset $S, \operatorname{Cay}\left(D_{2 n},\left\{b, a b, \ldots, a^{n-1} b\right\}\right)$ are the complete bipartite graph $K_{n, n}$ with eigenvalues $\lambda=0$ with multiplicity $2 n-2$ and $\lambda= \pm n$ with multiplicity 1. Therefore, the energy of the Cayley graphs of $D_{2 n}$ with respect to the subset $S$,

$$
E\left(C a y\left(D_{2 n},\left\{b, a b, \ldots, a^{n-1} b\right\}\right)\right)=(2 n-2)|0|+|n|+|-n|=2 n .
$$

The computation of the energy of Cayley graph with respect to the generating set $S=$ $\left\{b, a b, a^{2} b\right\}$ of dihedral groups of order $6, D_{6}$ is as shown in the following example.

\section{Example}

Let $D_{6}$ be the dihedral groups of order 6 , where $D_{6}=\left\langle a, b \mid a^{3}=b^{2}=1, b a b=a^{-1}\right\rangle$ and $S=\left\{b, a b, a^{2} b\right\}$ be the subset of $D_{6}$. The Cayley graph of $D_{6}$ with respect to the subset $S, C a y\left(D_{6},\left\{b, a b, a^{2} b\right\}\right)$ are the complete bipartite graph $K_{3,3}$. The eigenvalues of

$$
\operatorname{Cay}\left(D_{6},\left\{b, a b, a^{2} b\right\}\right)
$$

are 0 with multiplicity 4 and \pm 3 with multiplicity 1 . Then, the energy of the Cayley graphs of $D_{6}$ with respect to the subset $S, E\left(C a y\left(D_{6},\left\{b, a b, a^{2} b\right\}\right)\right)$ is 6 .

Proof Consider the dihedral groups of order $6, D_{6}=\left\langle a, b \mid a^{3}=b^{2}=1, b a b=a^{-1}\right\rangle$ and $S=$ $\left\{b, a b, a^{2} b\right\}$ be the subset of $D_{6}$. Then, by Definition 2, the vertex $x$ is connected to $s x$, where $s \in S$.

$$
\begin{array}{ll}
1-b \text { since } b .1=b & a-a^{2} b \text { since } b a=a^{-1} b^{-1}=a^{2} b \\
1-a b \text { since } a b .1=a b & a-b \text { since } a b a=a^{3} b=b \\
1-a^{2} b \text { since } a^{2} b .1=a^{2} b & a-a b \text { since } a^{2} b a=a^{4} b=a b \\
a^{2}-a b \text { since } b a^{2}=a^{2} b a=a^{4} b=a b & b-1 \text { since } b^{2}=1 \\
a^{2}-a^{2} b \text { since } a b a^{2}=b a=a^{2} b & b-a \text { since } a b^{2}=a \\
a^{2}-b \text { since } a^{2} b a^{2}=a b a=a^{3} b=b & b-a^{2} \text { since } a^{2} b^{2}=a^{2} \\
a b-a^{2} \text { since } b a b=a^{-1}=a^{2} & a^{2} b-a \text { since } b a^{2} b=a^{2} b a b=a^{4} b^{2}=a \\
a b-1 \text { since } a b a b=a^{3}=1 & a^{2} b-a^{2} \text { since } a b a^{2} b=a^{2} \\
a b-a \text { since } a^{2} b a b=a^{4}=a & a^{2} b-1 \text { since } a^{2} b a^{2} b=a^{3}=1
\end{array}
$$

The connected elements form the $\operatorname{Cay}\left(D_{6},\left\{b, a b, a^{2} b\right\}\right)=K_{3,3}$ as illustrated in Figure 1 . 


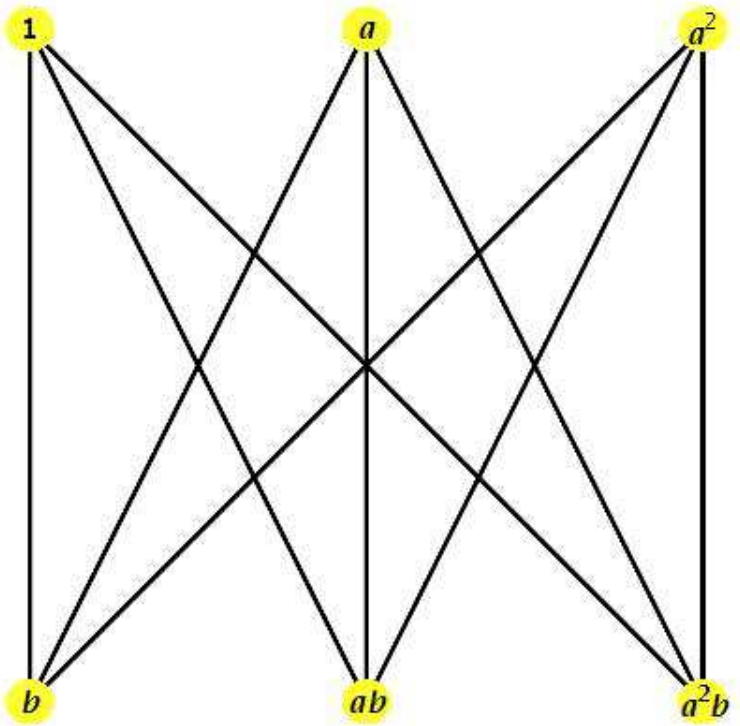

Figure 1: $\operatorname{Cay}\left(D_{6},\left\{b, a b, a^{2} b\right\}\right)=K_{3,3}$

By the definition of adjacency matrix,

$$
A\left(\operatorname{Cay}\left(D_{6},\left\{b, a b, a^{2} b\right\}\right)\right)=\left[\begin{array}{cccccc}
0 & 0 & 0 & 1 & 1 & 1 \\
0 & 0 & 0 & 1 & 1 & 1 \\
0 & 0 & 0 & 1 & 1 & 1 \\
1 & 1 & 1 & 0 & 0 & 0 \\
1 & 1 & 1 & 0 & 0 & 0 \\
1 & 1 & 1 & 0 & 0 & 0
\end{array}\right] .
$$

From the adjacency matrix, the characteristic polynomials found is $f(\lambda)=\lambda^{6}-9 \lambda^{4}$, which gives the eigenvalues $\lambda=0$ with multiplicity 4 and $\lambda= \pm 3$ with multiplicity 1 . By using the generalization of spectrum of a complete bipartite graph, the eigenvalues can be found as $\operatorname{Spec}\left(K_{n, n}\right)=\left\{ \pm \sqrt{n^{2}}, 0^{2 n-2}\right\}=\left\{ \pm \sqrt{3^{2}}, 0^{2(3)-2}\right\}=\left\{ \pm 3,0^{4}\right\}$. Therefore,

$$
E\left(C a y\left(D_{2 n},\left\{b, a b, a^{2} b\right\}\right)\right)=4|0|+1|3|+1|-3|=6 .
$$

\section{Conclusion}

For conclusion, it has been found that the energy of the Cayley graphs of the dihedral groups of order $2 n$, where $n \geq 3$ with respect to the subset $S=\left\{b, a b, \ldots, a^{n-1} b\right\}$ are $2 n$.

\section{Acknowledgments}

The authors would like to express their appreciation for the support of the sponsor; Ministry of Higher Education (MOHE) Malaysia and Research Management Centre (RMC), Universiti Teknologi Malaysia (UTM) Johor Bahru for the financial funding through the Research University Grant (GUP) Vote No. $13 \mathrm{H} 79$ and 13J82. The first author would also like to express her appreciation to UTM for the financial support under Zamalah Scholarship. 


\section{References}

[1] Woods, C. My Favorite Application Using Graph Eigenvalues: Graph Energy. 2013.

[2] Li, X., Shi, Y. and Gutman, I. Graph Energy. Springer Science \& Business Media. 2012.

[3] Bapat, R. B. and Pati, S. Energy of a Graph is Never an Odd Integer. 2004.

[4] Pirzada, S. and Gutman, I. Energy of a graph is never the square root of an odd integer. Applicable Analysis and Discrete Mathematics. 2008. 118-121.

[5] Foster-Greenwood, B. and Kriloff, C. Spectra of Cayley graphs of complex reflection groups. Journal of Algebraic Combinatorics. 2016. 44(1): 33-57.

[6] Liu, X. and Li, B. Distance powers of unitary Cayley graphs. Applied Mathematics and Computation. 2016. 272-280.

[7] Wang, C., and Xu, M. Non-normal one-regular and 4-valent Cayley graphs of dihedral groups $D_{2 n}$. European Journal of Combinatorics. 2006. 27(5): 750-766.

[8] Kwak, J. H., and Oh, J. M. One-regular Normal Cayley Graphs on Dihedral Groups of Valency 4 or 6 with Cyclic Vertex Stabilizer. Acta Mathematica Sinica. 2006. 22(5): 1305-1320.

[9] Kwak, J. H., Kwon, Y. S., and Oh, J.M. Infinitely many one-regular Cayley graphs on dihedral groups of any prescribed valency. Journal of Combinatorial Theory, Series B. 2008. 98(3): 585-598.

[10] Kim, D. S., Kwon, Y. S., and Lee, J. U. A classification of prime-valent regular Cayley maps on abelian, dihedral and dicyclic groups. Bulletin of the Korean Mathematical Society. 2010. 47(1): 17-27.

[11] Dehmer, M. Li, X., and Shi, Y. Connections between generalized graph entropies and graph energy. Complexity. 2015. 21: 35-41.

[12] Wu, H., Zhang, Y., Chen, W., and Mu, Z. Comparative analysis of protein primary sequences with graph energy. Physica A: Statistical Mechanics and its Applications. 2015. 437: 249-262.

[13] Daianu, M., Mezher, A., Jahanshad, N., Hibar, D. P., Nir, T. M., Jack, C. R., Weiner, M. W., Bernstein, M. A., Thompson, P. M. Spectral graph theory and graph energy metrics show evidence for the Alzheimers disease disconnection syndrome in APOE-4 risk gene carriers. Proc. IEEE Int. Symp. Biomed Imaging 2015. 2015. 458-461.

[14] Rotman, J. J. Advanced Modern Algebra. USA: Pearson Education. 2008.

[15] Beineke, L. W. and Wilson, R. J. Topics in Algebraic Graph Theory. USA: Cambridge University Press. 2004.

[16] Brouwer, A.E. and Haemers, W.H. Spectra of Graphs. Springer Science \& Business Media. 2011.

[17] Bapat, R. B. Graphs and Matrices. New York: Springer. 2010. 